3

\title{
Automated identification of piglet brain tissue from MRI images using Region-Based Convolutional Neural Networks
}

\author{
Kayla L. Stanke ${ }^{1}$, Ryan J. Larsen ${ }^{1, *}$, Laurie Rund ${ }^{1}$, Brian J. Leyshon ${ }^{2}$, Allison Y. Louie ${ }^{3}$, Andrew J. \\ Steelman ${ }^{1,3,4,5}$ \\ ${ }^{1}$ Department of Animal Sciences, University of Illinois Urbana-Champaign \\ ${ }^{2}$ Abbott Nutrition, Discovery Research, Columbus, Ohio \\ ${ }^{3}$ Division of Nutritional Sciences, University of Illinois Urbana-Champaign \\ ${ }^{4}$ Neuroscience Program, University of Illinois Urbana-Champaign \\ ${ }^{5}$ Carl R. Woese Institute for Genomic Biology, University of Illinois Urbana-Champaign
}

*Corresponding author

Email: 1arsen@illinois.edu

\section{Abstract}

Magnetic Resonance Imaging is an important tool for characterizing volumetric changes of the piglet brain during development. Typically, an early step of an imaging analysis pipeline is brain extraction, or skull stripping. Brain extractions are usually performed manually; however, this approach is time-intensive and can lead to variation between brain extractions when multiple raters are used. Automated brain extractions are important for reducing the time required for analyses and improving the uniformity of the extractions. Here we demonstrate the use of Mask R-CNN, a Region-based Convolutional Neural Network (R-CNN), for automated brain extractions of piglet brains. We validate our approach using Nested Cross-Validation on six sets of training/validation data drawn from 32 pigs. Visual inspection of the extractions shows acceptable accuracy, and 
25 Dice coefficients are in the range of 0.95-0.97. These results demonstrate that R-CNNs provide a

26 viable tool for skull stripping of piglet brains.

\section{Introduction}

28 Piglets are an important translational model for measuring the effect of nutrition on brain

29 development. Not only do piglet brain stages of development correlate with human infant

30 development, but their nutritional requirements are also comparable (1). Magnetic resonance

31 imaging (MRI) is an important technique for obtaining non-invasive measurements of brain

32 volumes. An early step in volumetric analysis is the identification or "extraction" of the brain from

33 the surrounding tissue. Manual tracing has been the gold standard for brain extraction and is

34 performed by creating an outline that separates the surrounding skull, muscles, tissues, and fat

35 from the brain (1-6). However, the method is not ideal when working with large data sets because

36 it is time intensive and is subject to inconsistencies between raters/evaluators. Automated brain

37 extraction techniques are needed to overcome these limitations. However, reports of automated

38 extractions of pig brains are limited. A graph theory approach that makes use of prior information

39 of anatomical structures has been used to perform automated extraction of piglet brains (7).

40 However, deep learning technologies offer the possibility of automating the training of anatomical

41 structures to enable brain extractions (8-10). A U-Net model trained on humans, non-human

42 primates, and 3 pig scans has been shown to successfully perform brain extractions on 2 pig scans

43 (11). An alternative segmentation tool is Mask R-CNN, a Region-based Convolutional Neural

44 Network (R-CNN). This tool has been used to create a mouse brain atlas that is generalizable

45 across developmental ages and imaging modalities (12). These results suggest that Mask R-CNN 
may be effective for piglet brain extraction. Here we demonstrate the use of Mask R-CNN for

47 automated brain extractions of piglet brains.

\section{Methods}

\section{Animals and Care Practices}

All animal care and handling procedures were approved by the University of Illinois Institutional

Animal Care and Use Committee (Protocol \#18256) and were in accordance with federal

52 guidelines. Male and female average-for-gestational age, Yorkshire crossbred, full-term, naturally-

53 delivered piglets were obtained from University of Illinois Swine Farm at postnatal day 2 to allow

54 for colostrum consumption. All piglets remained intact but did undergo routine processing on the

55 farm including iron dextran (Henry Schein Animal Health, Dublin, OH, USA) and antibiotic

56 injection (EXCEDE®, Zoetis, Parsippany, NJ 07054, USA) per routine farm practice and

57 according to label. Four groups of piglets were placed individually into a caging system under

58 standard conditions as described in a previous publication (13) and randomly assigned to five diet

59 treatment groups. The control group of piglets remained with the sow until day 28 of age with free

60 access to suckle and were weighed daily. The current study does not distinguish between diet

61 groups.

62 Sow-raised piglets were handled daily, as were the artificially raised animals to diminish

63 differences between the two types of rearing. After arrival at the Edward R. Madigan Laboratory

64 (ERML) Animal Facility, experimental piglets received one dose of antibiotic: the first of two

65 cohorts received Spectraguard ${ }^{\mathrm{TM}}$ (Bimeda, Inc, Oakbrook Terrace, IL 60181) on postnatal day 2

66 and the second cohort received Baytril@ (Bayer healthcare LLC Shawnee Mission, KS 66201) on

67 postnatal day 4. Additional doses of antibiotic were administered during the experiment only under 
the direction of the staff veterinarian. Animals were individually housed in racks of metabolism cages specifically designed to artificially rear neonatal piglets under constant 12 -h light/dark cycles. Piglet housing at ERML was as follows: space allowance for individual piglets was 30" deep, 23" wide, and 18.5" high, providing 4.8 square feet of floor space per piglet. Each piglet was supplied with a heating pad, toy, and blanket. Room temperatures were kept at $85-95^{\circ} \mathrm{F}$ using space heaters. Animals were also offered water or BlueLite ${ }^{\circledR}$ electrolyte solution ad libitum.

74 Cages and heating pads were disinfected, and toys and blankets replaced daily. Animal care 75 protocols were in accordance with National Institutes of Health Guidelines for Care and Use of 76 Laboratory Animals and were approved by the University of Illinois Laboratory Animal Care and 77 Use Committee.

\section{MRI Acquisition}

MRI data was acquired using 3 T Prisma scanner (Siemens, Erlangen) housed at the Biomedical

84 (Rapid Biomed, Rimpar). During scanning, the respiration rate, heart rate and blood oxygen levels were monitored using a LifeWindow LW9x monitor (Digicare, Boynton Beach, FL).

87 size $=0.6 \times 0.6 \times 0.6 \mathrm{~mm}^{3}, \mathrm{FOV}=173 \times 173 \mathrm{~mm}^{2}, 256$ slices, GRAPPA - GeneRalized

88 Autocalibrating Partial Parallel Acquisition - acceleration factor R = 2; TR = $2060 \mathrm{ms,} \mathrm{TI} \mathrm{=} 1060$ $\mathrm{ms}$, flip angle $=9^{\circ}$, for an overall scan time of 5:21 $\mathrm{min}$ ). 
91 Manual brain extraction was facilitated by first performing a rigid alignment of $\mathrm{T}_{1}$-weighted

92 images from all piglets to the brain atlas (14). This was done using SPM12 imaging analysis software. First, we performed a manual rotation to approximately align the $\mathrm{T}_{1}$-weighted images with 28-day piglet template (14), without resampling. We then used the "coregistration" function of SPM12 to further align the $\mathrm{T}_{1}$-weighted images to the template, again without resampling. The resulting alignment was accurate for all piglets, even though it was performed without first performing brain extraction, as shown by a representative image in Figure 1.
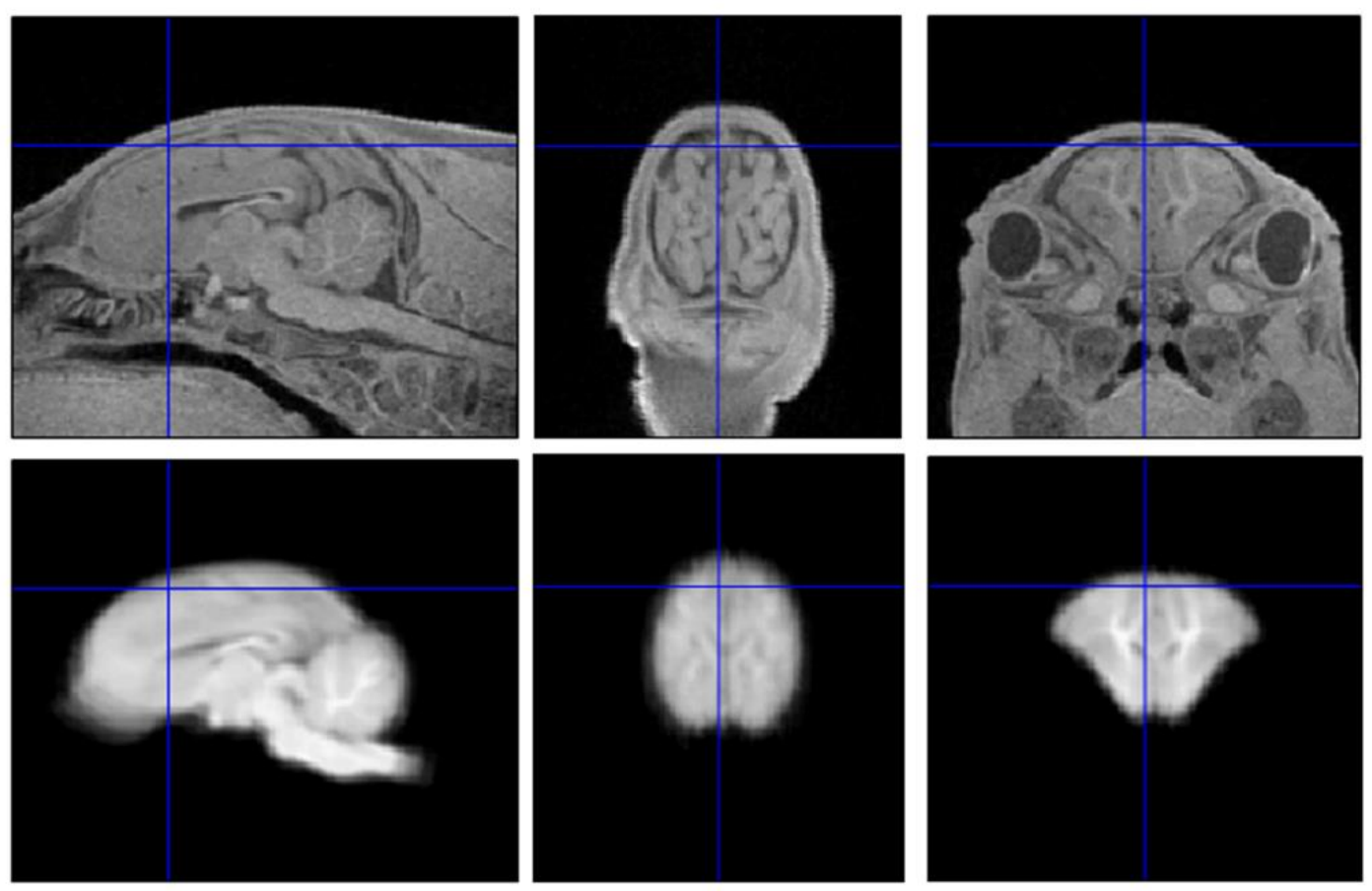

Figure 1. Results of coregistration of piglet brain to the average brain template. The top row 
slices. The consistency of two sets of images confirms a good approximate alignment of the $T_{1^{-}}$ weighted image with the average brain template.

An atlas-based brain mask was then resampled into the native space of each piglet, providing initial estimates of the brain masks for each piglet. These initial estimates were then modified to create individualized brain masks using Slicer3D, as shown in Figure 2. Most revisions were done in the sagittal plane, but all three orthogonal views were reviewed and modified for improved precision. All extractions were performed by one rater to minimize variability.

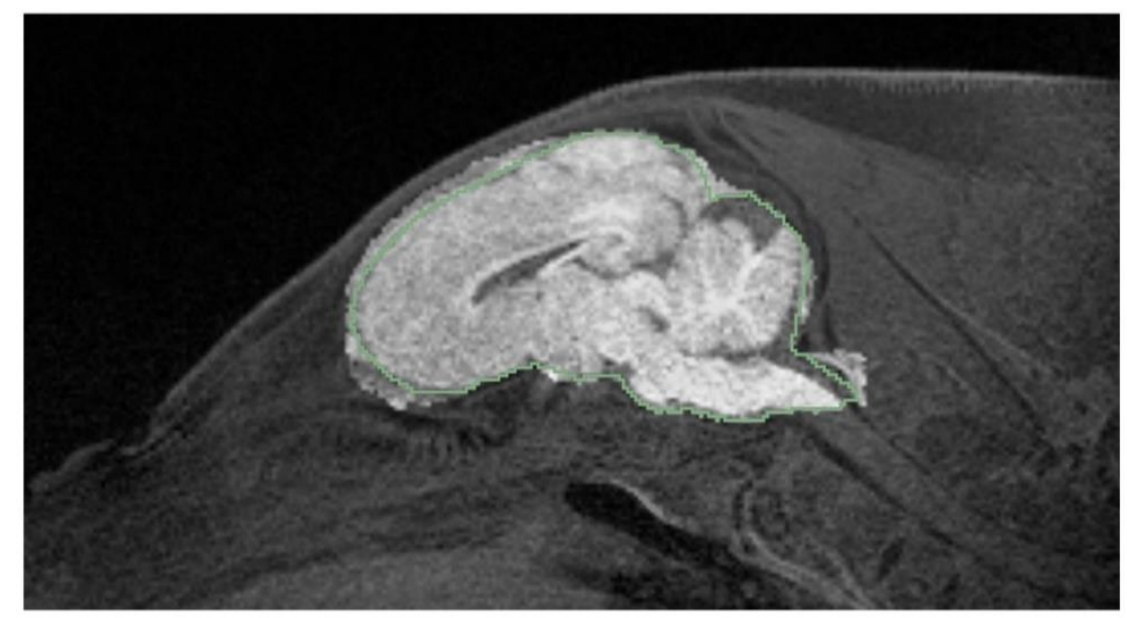

Figure 2. Demonstration of the creation of manually-defined brain mask via modification of the template brain mask. A representative $T_{1}$-weighted image is overlayed with the template brain mask, creating a brighter region. The green outline shows the manual-designated brain mask. The manual brain mask was created by editing the template brain mask for each

114 individual piglet. 
(16). Our model uses a Tensor Flow 2.4.0 implementation of Mask R-CNN (15), with feature extractor Faster R-CNN (17). Faster R-CNN utilizes a Region Proposal Network (RPN) to select object proposals from a backbone, which for our study was generated using a combined ResNet101 and Feature Pyramid Network (FPN) (18).

Training was performed using a single NVIDIA GeForce GTX 1070 Ti GPU, with NVIDIA developer driver 465.21, CUDA 11.0, and CUDNN library 8.0.4. The network was trained with a cosine decay learning rate of 0.008 and a momentum optimizer value of 0.9 , and a batch size of two images, or slices, per iteration. We performed 200,000 iterations, or 48.8 epochs. The training and segmentation were performed only in 2D sagittal planes. During evaluation, predicted masks were binarized at a confidence parameter threshold of 0.5 . The masks created from the $2 \mathrm{D}$ slices were then combined into 3D datasets for final cleaning. This involved removing several small and spurious masks, typically occurring in slices that did not include brain. Cleaning was done by first using the Matlab function "bwconncomp" to identify all isolated masks, consisting of one or more connected voxels, where connected voxels are defined as those with touching faces. Then we discarded all by the largest mask, or brain mask.

\section{Validation}

Nested Cross-Validation was used to evaluate the performance of the training models. This method has been shown to produce unbiased performance estimates, even in small datasets (19). We randomly assigned each of the 32 pigs to one of six test groups. Four of the test groups consisted of five pigs, and two of the test groups consisted of six pigs. For each test group, a training model was generated using the remaining pigs, beginning with the same architecture which was naïve to the test images. Validation of the test groups was performed by comparing the machine-generated masks with the manually generated masks by visual inspection, by computing Dice coefficients 
141 for each pig, and by computing Pearson correlations between the manual and machine-generated

142 masks. Dice coefficients were calculated using the formula $2|X \cap Y| /(|X|+|Y|)$, where $|\ldots|$

143 indicates the number of voxels within the mask, $\mathrm{\cap}$ indicates the union, and $X$ and $\mathrm{Y}$ indicate the

144 manual and machine-generated masks.

\section{Results}

146 Visual inspection of the brain extractions reveals good accuracy of automatic brain extractions

147 (Figure 3). The six models were labelled with letters from A to F. We found that Model D failed

148 to identify the brain within several sagittal slices of one of the test pigs, as shown in Figure 4(a)

149 and (b). These slices included an unusually bright region in the subcutaneous fat layer near the

150 superior area of the head (see Figure 4(b)). This bright region was removed by manually outlining

151 it in one of the slices, and then removing the traced voxels from all the slices (see Figure 4(d)).

152 The modified structural images were then re-evaluated using the same model, producing a more

153 accurate brain mask (see Figure 4(c) and (d)). This brain mask was used for subsequent validation.

154 Dice coefficients from the 32 test scans were in the range of 0.95-0.97 (mean: 0.961, standard

155 deviation: 0.0036, see histogram in Figure 5). The Pearson correlation coefficient, $R$, of the

156 volumes, $V_{\text {manual }}$ and $V_{\text {machine }}$, of the manual and machine-generated masks was $\mathrm{R}=0.90$, with

$157 \mathrm{p}<0.001$ (see Figure 6). 


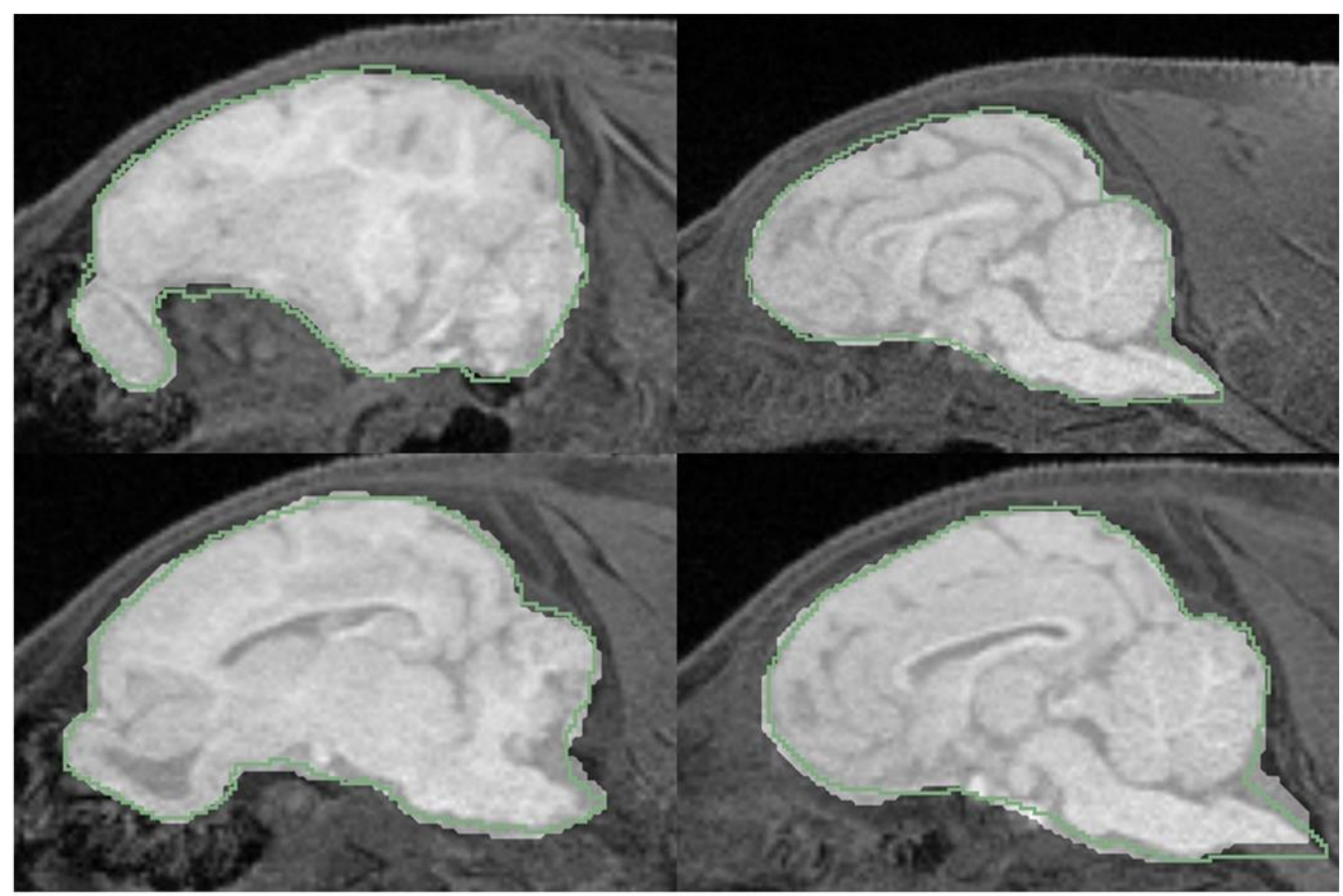

Figure 3. Sample extractions from two piglets. The overlay that creates a brighter region indicates machine extractions, and the green outline shows the manual brain masks. The top

B. 

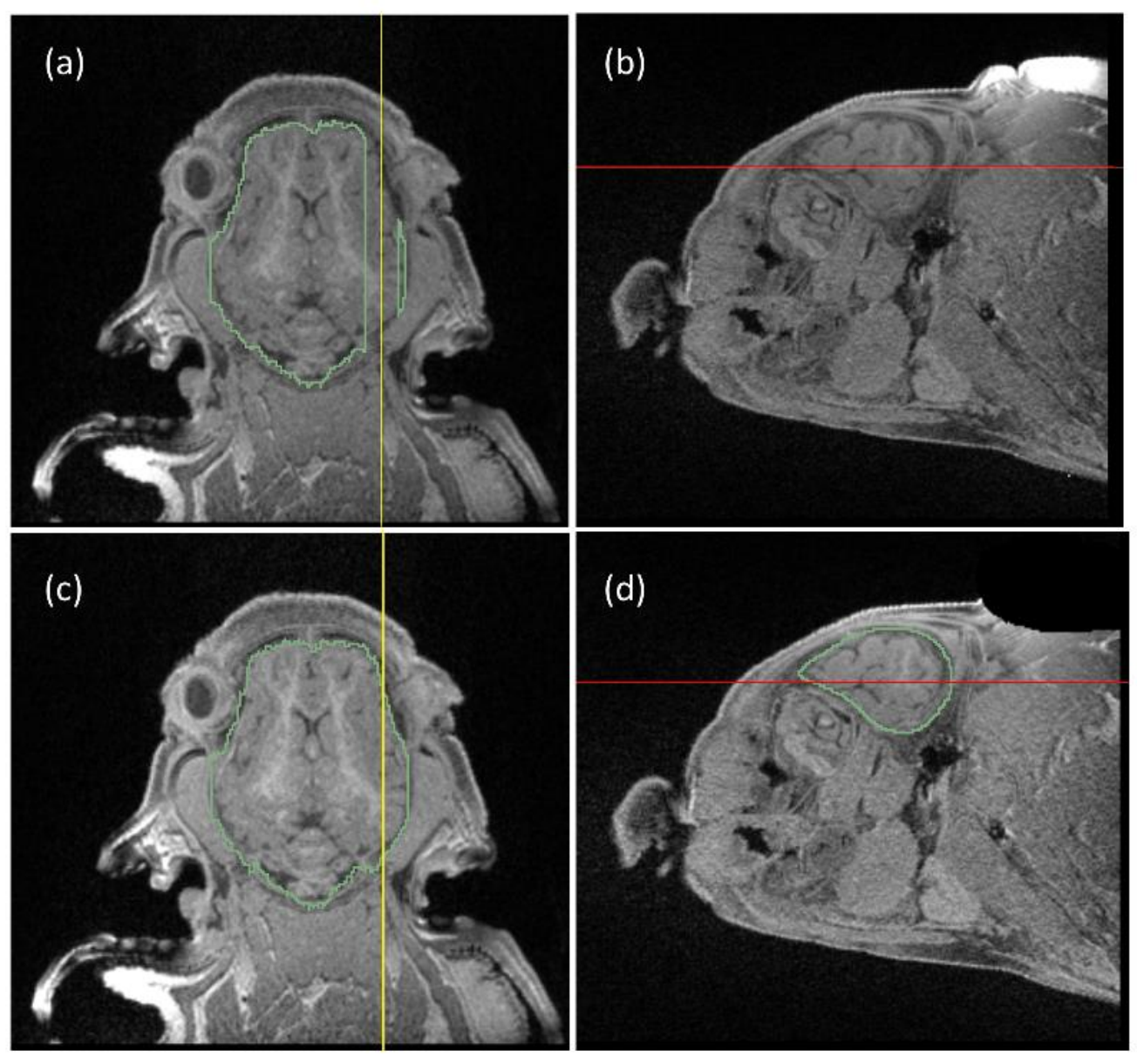

Figure 4. A segmentation error generated by Model D that was subsequently corrected. Panels (a) and (b) show the inaccurate mask, indicated by green lines, and panels (c) and (d) show the mask generated by the same model after image modification, with the same views and the same slices. Axial slices are shown in (a) and (c); sagittal slices are shown in (b) and (d). The yellow lines in the axial views, (a) and (c), show the location of the sagittal view and the red lines in the sagittal views, (b) and (d), show the location of the axial views. For the inaccurate mask there are several sagittal slices in which no brain regions were identified, as shown in (a). These

171 slices included a bright region in the fat layer of the superior region of the head, as shown in 172 (b). The bright region was manually traced and removed from the same voxels of all sagittal 
bioRxiv preprint doi: https://doi.org/10.1101/2021.07.23.453526; this version posted November 29, 2021. The copyright holder for this preprint (which was not certified by peer review) is the author/funder, who has granted bioRxiv a license to display the preprint in perpetuity. It is made available under aCC-BY 4.0 International license.

173 slices as shown in (d). A re-evaluation of the edited images using the same model, Model D,

174 produced an accurate brain extraction, as shown in (c) and (d).

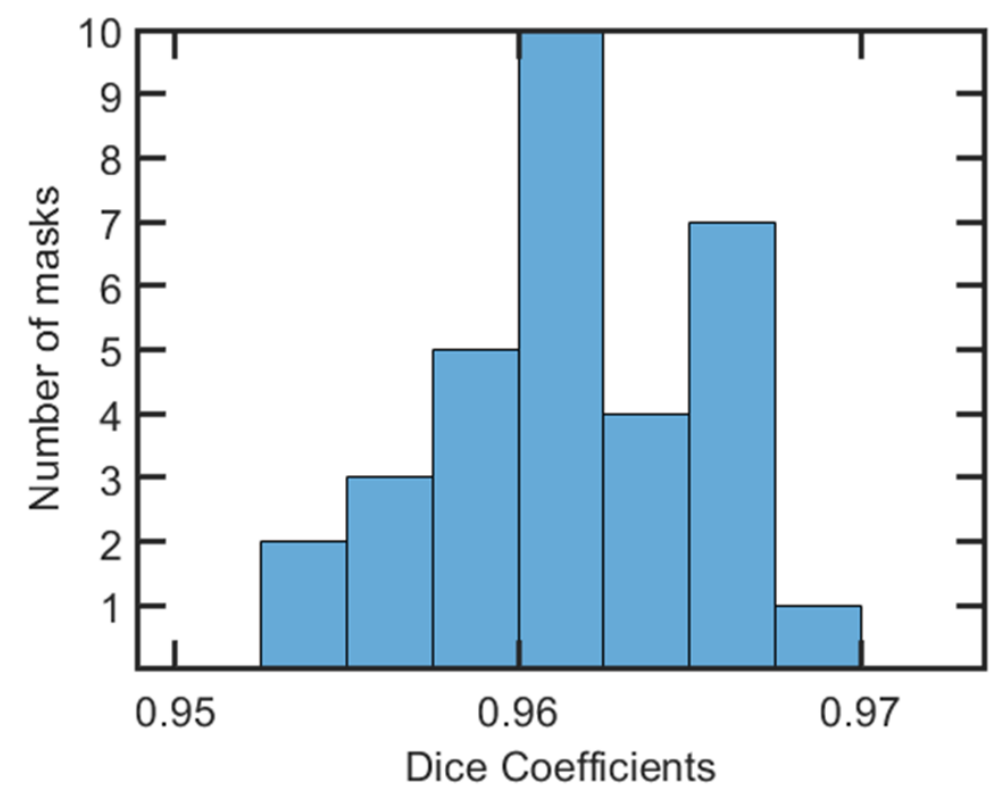

176 Figure 5. Histogram of Dice coefficients calculated from each of the 32 pigs. 


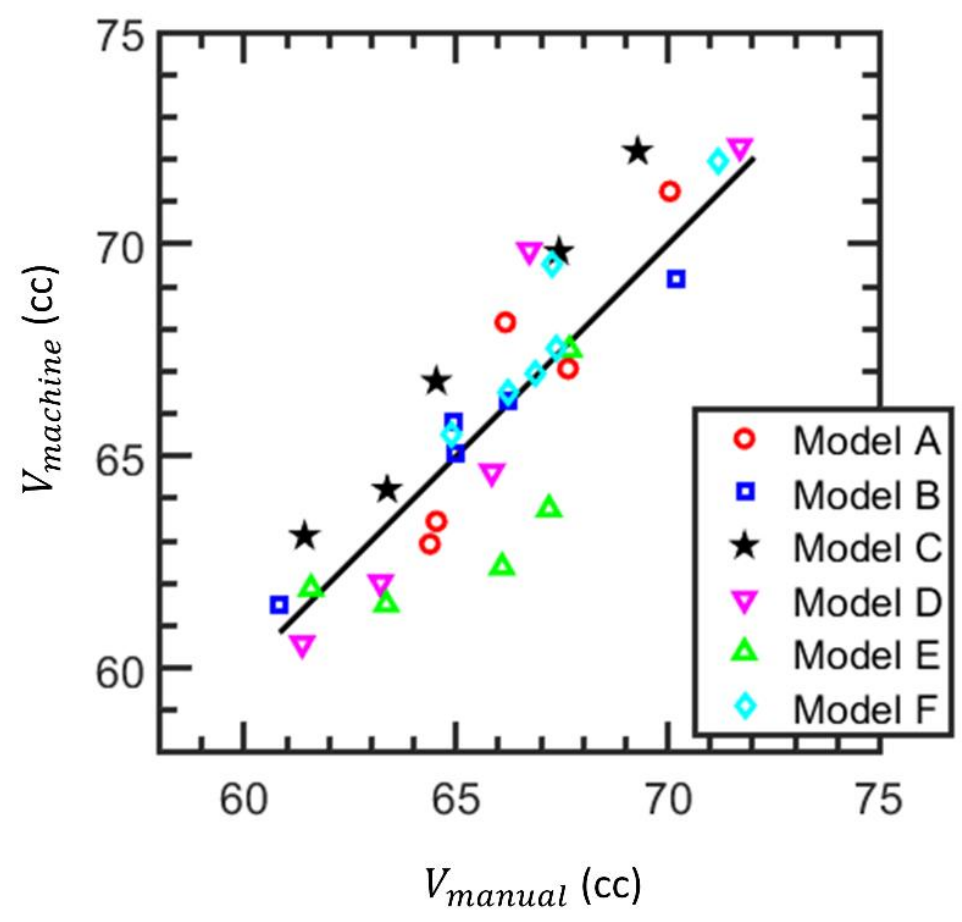

179 Figure 6. The consistency of brain volumes, $V_{\text {manual }}$ and $V_{\text {machine }}$, from manual and machine brain extractions, respectively. The solid line indicates unity and the colors denote the models used. Brain volume units are cubic centimeters.

\section{Discussion}

We have shown that Mask R-CNN trained on manually generated masks can be used to perform brain tissue within several slices. This problem was eliminated when the evaluation was repeated after the removal of an unusually bright region of subcutaneous fat was removed from the images. 
189

190

191

192

193

194

195

performing a bias field correction before training. This example underscores the importance of visual checks of machine performance.

Dice coefficients were $>0.95$; these are similar to values that have been achieved by neural networks for the skull-stripping of non-human primates (11) and rodents (20).

The use of automated brain extraction has the potential to reduce analysis time because it requires minimal supervision. This process is scalable to a high number of piglets, avoiding complications and inconsistencies that might arise from having multiple raters perform manual brain extractions. Improvements to our approach could be implemented in a variety of ways. Training with a larger sample size is expected to increase performance and accuracy of machine learning algorithms (21). Performance may also be improved by hyperparameter tuning and optimization $(22,23)$. Improved performance might have been obtained by using 3D Mask R-CNN; however we employed 2D Mask R-CNN to obtain shorter training times (24). Performance may also be improved by using quantitative imaging techniques, such as MT saturation $(25,26)$, to improve the contrast between brain and non-brain tissues. Further research is required to access whether network architectures such as U-Net (27), may improve upon results obtained with Mask R-CNN.

The effectiveness of Mask R-CNN for performing piglet brain extractions implies that it may be a useful tool for segmenting sub-regions of the brain. Further research is needed to assess whether such an approach may compliment or improve upon existing methods for volumetric analysis of piglet brain MRI data (4, 28, 29).

\section{Acknowledgments}

The study was funded by Abbott Nutrition, www.abbott.com, grant number 00490326 to A.S. The funder aided with study design, data analysis, decision to publish, and preparation of the 
manuscript. We thank the staff members of the Biomedical Imaging Center at the Beckman

\section{Author contributions}

214 KLS: Conceptualization, Data Curation, Formal Analysis, Investigation, Methodology, Software,

215 Validation, Visualization, Writing - Original Draft Preparation, Writing-Review \& Editing RJL:

216 Conceptualization, Data Curation, Formal Analysis, Funding Acquisition, Investigation,

217 Methodology, Project Administration, Resources, Software, Supervision, Validation,

218 Visualization, Writing - Original Draft Preparation, Writing-Review \& Editing AL: Investigation,

219 Resources BJL: Conceptualization, Funding Acquisition, Investigation, Project Administration,

220 Supervision, Writing-Review \& Editing LR: Funding Acquisition, Investigation, Project

221 Administration, Resources, Supervision AS: Funding Acquisition, Supervision, Writing-Review

$222 \quad \&$ Editing

223

\section{Data Sharing}

Processing scripts are available at https://github.com/kaylals2/Piglet_RCNN.

225

226

227

228

229

230

231

232

233

234

235

236

237

238

239

240

241

\section{References}

1. Mudd AT, Dilger RN. Early-Life Nutrition and Neurodevelopment: Use of the Piglet as a Translational Model. Advances in Nutrition. 2017;8(1):92-104.

2. Bokde AL, Teipel SJ, Schwarz R, Leinsinger G, Buerger K, Moeller T, et al. Reliable manual segmentation of the frontal, parietal, temporal, and occipital lobes on magnetic resonance images of healthy subjects. Brain Res Brain Res Protoc. 2005;14(3):135-45.

3. Conrad MS, Dilger RN, Nickolls A, Johnson RW. Magnetic resonance imaging of the neonatal piglet brain. Pediatr Res. 2012;71(2):179-84.

4. Gan H, Zhang Q, Zhang H, Chen Y, Lin J, Kang T, et al. Development of new population-averaged standard templates for spatial normalization and segmentation of MR images for postnatal piglet brains. Magn Reson Imaging. 2014;32(10):1396-402.

5. Mudd AT, Alexander LS, Berding K, Waworuntu RV, Berg BM, Donovan SM, et al. Dietary Prebiotics, Milk Fat Globule Membrane, and Lactoferrin Affects Structural Neurodevelopment in the Young Piglet. Front Pediatr. 2016;4:4.

6. Leyshon BJ, Radlowski EC, Mudd AT, Steelman AJ, Johnson RW. Postnatal Iron Deficiency Alters Brain Development in Piglets. J Nutr. 2016;146(7):1420-7. 
7. Durandeau AF, J.-B.; Bloch, I.; Mazerand, E.; Menei, P.; Montero-Menei, C.; Dinomais, M. , editor Structural information and (hyper)graph matching for MRI piglet brain extraction. International Conference on Pattern Recognition Systems; 2019 8-10 July 2019; Tours, France.

8. Akkus Z, Galimzianova A, Hoogi A, Rubin DL, Erickson BJ. Deep Learning for Brain MRI Segmentation: State of the Art and Future Directions. J Digit Imaging. 2017;30(4):449-59.

9. Hurtz S, Chow N, Watson AE, Somme JH, Goukasian N, Hwang KS, et al. Automated and manual hippocampal segmentation techniques: Comparison of results, reproducibility and clinical applicability. Neuroimage Clin. 2019;21:101574.

10. Iqbal A, Khan R, Karayannis T. Developing a brain atlas through deep learning. Nature Machine Intelligence 2019;1:277-87.

11. Wang X, Li XH, Cho JW, Russ BE, Rajamani N, Omelchenko A, et al. U-net model for brain extraction: Trained on humans for transfer to non-human primates. Neuroimage. 2021;235:118001.

12. Iqbal A, Khan R, Karayannis T. Developing a brain atlas through deep learning. Nat Mach Intell. 2019;1(6):277-87.

13. Rytych JL, Elmore MR, Burton MD, Conrad MS, Donovan SM, Dilger RN, et al. Early life iron deficiency impairs spatial cognition in neonatal piglets. J Nutr. 2012;142(11):2050-6.

14. Conrad MS, Sutton BP, Dilger RN, Johnson RW. An in vivo three-dimensional magnetic resonance imaging-based averaged brain collection of the neonatal piglet (Sus scrofa). PLoS One. 2014;9(9):e107650.

15. He KM, Gkioxari G, Dollar P, Girshick R. Mask R-CNN. leee I Conf Comp Vis. 2017:2980-8.

16. Lin T-Y, Maire M, Belongie S, Hays J, Perona P, Ramanan D, et al., editors. Microsoft COCO: Common Objects in Context2014; Cham: Springer International Publishing.

17. Ren SQ, He KM, Girshick R, Sun J. Faster R-CNN: Towards Real-Time Object Detection with Region Proposal Networks. leee T Pattern Anal. 2017;39(6):1137-49.

18. Lin TY, Dollar P, Girshick R, He KM, Hariharan B, Belongie S. Feature Pyramid Networks for Object Detection. Proc Cvpr leee. 2017:936-44.

19. Vabalas A, Gowen E, Poliakoff E, Casson AJ. Machine learning algorithm validation with a limited sample size. PLoS One. 2019;14(11):e0224365.

20. Hsu LM, Wang S, Ranadive P, Ban W, Chao THH, Song S, et al. Automatic Skull Stripping of Rat and Mouse Brain MRI Data Using U-Net. Front Neurosci-Switz. 2020;14.

21. Jollans L, Boyle R, Artiges E, Banaschewski T, Desrivieres S, Grigis A, et al. Quantifying performance of machine learning methods for neuroimaging data. Neuroimage. 2019;199:351-65.

22. Yang L, Shami A. On hyperparameter optimization of machine learning algorithms: Theory and practice. Neurocomputing. 2020;415:295-316.

23. Wu J, Chen X-Y, Zhang H, Xiong L-D, Lei H, Deng S-H. Hyperparameter Optimization for Machine Learning Models Based on Bayesian Optimizationb. Journal of Electronic Science and Technology. 2019;17(1):26-40.

24. Jeong J, Lei Y, Kahn S, Liu T, Curran WJ, Shu HK, et al. Brain tumor segmentation using 3D Mask R-CNN for dynamic susceptibility contrast enhanced perfusion imaging. Phys Med Biol. 2020;65(18).

25. Helms G, Draganski B, Frackowiak R, Ashburner J, Weiskopf N. Improved segmentation of deep brain grey matter structures using magnetization transfer (MT) parameter maps. Neuroimage. 2009;47(1):194-8.

26. Helms G, Dathe H, Kallenberg K, Dechent P. High-resolution maps of magnetization transfer with inherent correction for RF inhomogeneity and T1 relaxation obtained from 3D FLASH MRI. Magn Reson Med. 2008;60(6):1396-407.

27. Ronneberger $\mathrm{O}$, Fischer P, Brox T, editors. U-Net: Convolutional Networks for Biomedical Image Segmentation2015; Cham: Springer International Publishing.

28. Ashburner J, Friston KJ. Unified segmentation. Neuroimage. 2005;26(3):839-51. 
bioRxiv preprint doi: https://doi.org/10.1101/2021.07.23.453526; this version posted November 29, 2021. The copyright holder for this preprint (which was not certified by peer review) is the author/funder, who has granted bioRxiv a license to display the preprint in perpetuity. It is made available under aCC-BY 4.0 International license.

290 29. Radlowski EC, Conrad MS, Lezmi S, Dilger RN, Sutton B, Larsen R, et al. A neonatal piglet model 291 for investigating brain and cognitive development in small for gestational age human infants. PLoS One. 292 2014;9(3):e91951. 